\title{
Estudio de un brote de intoxicación por alcohol metílico en Santa Fe de Bogotá, 1994
}

\author{
Marcela Varona ${ }^{1}$, Gloria Suárez ${ }^{2}$, Miguel Velásquez ${ }^{3}$, Pedro Morales ${ }^{4}$, Carmen Sánchez ${ }^{5}$
}

\begin{abstract}
Resumen
EI Laboratorio de Toxicología del Instituto Nacional de Medicina Legal y Ciencias Forenses (INML y CF) observó un incremento de muestras positivas para metanol en sangre, entre las necropsias medicolegales practicadas en junio de 1994. Por lo anterior, se llevó a cabo un estudio epidemiológico con el fin de establecer el diagnóstico, identificar los factores de riesgo y controlar y prevenir la presencia de nuevos brotes.
\end{abstract}

Se realizó un estudio descriptivo, se revisaron los protocolos de necropsia, los resultados de alcoholemia e historias clínicas de los casos y se aplicó un formulario de necropsia verbal. Se definió como caso mortal de intoxicación metílica (ITM) aquella defunción ocurrida durante junio de 1994, cuya necropsia se realizó en el INML y CF, con concentraciones de metanol superiores a $30 \mathrm{mg} / \mathrm{dL}$ y cuyo informe establecía intoxicación por metanol como causa básica de la defunción.

Se identificaron 14 personas fallecidas por ITM, siendo la totalidad de los casos indigentes. La función estadística scan $(\mathrm{p}=0,004)$ indicó que el exceso de defunciones de junio era estadísticamente significativo en relación con los meses previos. Doce de los casos recibieron atención médica y a cuatro se les diagnosticó ITM antes de su muerte. Todos los casos presentaron concentraciones de metanol en sangre entre 43 y 190,4 mg/dL.

Este es el primer conglomerado de intoxicación por metanol en indigentes que se describen en el país y plantea la necesidad de establecer políticas de salud pública dirigidas a la protección de indigentes y prevención de intoxicación por metanol.

\section{Summary}

The National Institute of Legal Medicine and Forensic Science's Toxicology Laboratory (Laboratorio de Toxicología del Instituto Nacional de Medicina Legal y Ciencias Forenses, INML y CF), observed an increase in positive samples for methanol in blood from medicolegal necropsies performed in June, 1994. Thus an epidemiological study was designed to establish diagnosis, to identify risk factors and to control and prevent new outbreaks.

A descriptive study was carried out, necrosy protocol, alcoholism results and clinical histories of such cases were revised and a verbal necropsy was made in response to a prepared

\footnotetext{
1 Grupo de Salud Ambiental, Instituto Nacional de Salud, Programa de Adiestramiento Avanzado en Epidemiología Aplicada INS-CDC.

${ }^{2}$ Grupo de Clínica Forense, Instituto Nacional de Medicina Legal y Ciencias Forenses, Programa de Adiestramiento Avanzado en Epidemiología Aplicada, INS-CDC.

${ }^{3}$ Grupo de Química Forense, Instituto Nacional de Medicina Legal y Ciencias Forenses.

${ }^{4}$ Grupo de Patología Forense, Instituto Nacional de Medicina Legal y Ciencias Forenses.

${ }^{5}$ Asesora, Programa de Epidemiología Aplicada
} 
questionnaire. Cases of fatal methyl poisoning (FMP) were defined as being those deaths which ocurred during June, 1994, whose necropsy was carried out at INML y CF, having methanol concentrations greater than $30 \mathrm{mg} / \mathrm{dL}$ and whose report established methanol poisoning as the basic cause of death.

Fourteen people died from FMP, the total number of cases being amongst destitute people. The 'scan' statistical function $(p=0,004)$ showed that the excess of deaths in June, 1994, was statistically significant in relation to previous months. Twelve of the cases received medical attention and four were diagnosed as suffering from FMP before their deaths. All cases presented methanol concentrations in blood between 43 and $190.4 \mathrm{mg} / \mathrm{dL}$.

This is the first methanol poisoning conglomeration amongst destitute people which has been described in the country and has shown the necessity of establishing public health policies directed towards protection of destitute people and the prevention of methanol poisoning.

El alcohol metílico es un tóxico que ocasiona una alta mortalidad y, también, secuelas invalidantes cuando se ingiere (1-3). La causa más frecuente de intoxicación se presenta por la ingestión de bebidas alcohólicas adulteradas. En la literatura mundial, aparecen consignados muchos accidentes ocasionados por metanol como el ocurrido en Atlanta en 1951, en el que se intoxicaron 323 personas de las cuales fallecieron 41 (4); en Lexington (Kentucky) en 1960 con 18 intoxicados (5) y en Michigan en mayo de 1979 con 46 intoxicaciones, presentándose tres defunciones (6).

En Colombia, frecuentemente se presentan casos aislados, pero, también brotes como el ocurrido en septiembre de 1989 donde se intoxicaron 31 personas de las cuales 21 fallecieron. De las personas que sobrevivieron, cinco presentaron ceguera además de graves secuelas neurológicas (7).

Durante junio de 1994, el Laboratorio de Toxicología del Instituto Nacional de Medicina Legal y Ciencias Forenses (INML y CF), regional Bogotá, observó un incremento en el número de muestras positivas para alcohol metílico entre las personas fallecidas a las que se les practicó necropsia medicolegal. El análisis de etanol y metanol en sangre se realiza de rutina a todas las personas fallecidas por muerte violenta y a quienes por criterio del patólogo se requiera. De las 698 necropsias practicadas durante este mes, se pudo establecer como causa de la muerte, la intoxicación por alcohol metílico en 14 personas fallecidas. Entre agosto de 1993 a julio de 1994, se registraron 51 personas fallecidas por esta intoxicación en la misma institución. Su distribución se muestra en la figura 1.

Ante este hecho, el Grupo de Química Forense del INML y CF contactó al Grupo de Clínica Forense de la misma institución y al Programa de Epidemiología Aplicada del Instituto Nacional de Salud con el fin de estudiar el brote presentado con los objetivos que se citan a continuación:

1. Confirmar el diagnóstico de intoxicación metílica en los casos mortales.

2. Confirmar la existencia de un brote.

3. Identificar factores de riesgo en intoxicación por alcohol metílico.

4. Descartar una fuente de exposición común.

5. Identificar medidas de control y prevención de las defunciones por alcohol metílico.

\section{Materiales y métodos}

Se trata de un estudio descriptivo en el que se estudiaron los casos correspondientes a indigentes residentes en Bogotá quienes fallecieron por intoxicación con metanol en junio de 1994. Las variables analizadas fueron: edad, sexo, día y lugar del fallecimiento, zona donde habitualmente permanecían, estrato social, manifestaciones clínicas, antecedentes de ingestión de alcohol y de estupefacientes, concentraciones de alcohol etílico y metílico y hallazgos de necropsia forense, entre otros.

La fuente de datos estuvo constituida por los protocolos de necropsia del archivo del Grupo de 
Figura 1. Muertes asociadas con concentraciones elevadas de metanol en sangre, Bogotá, agosto de 1993 a julio 1994.

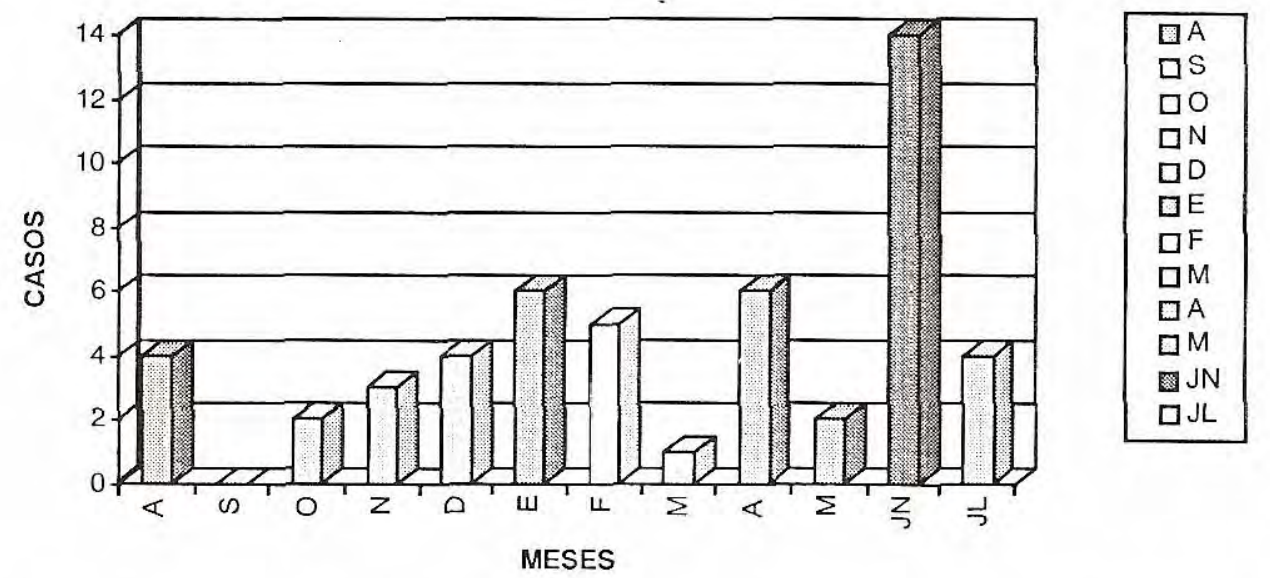

Patología Forense del INML y CF de cada uno de los casos cuya necropsia fue positiva para alcohol metílico, los cuales constan del acta de levantamiento del cadáver, el resumen de historia clínica, el informe de necropsia, el certificado de defunción y los resultados de laboratorio.

En los pacientes en quienes se pudo identificar el hospital o el centro de atención médica inmediata donde fueron atendidos, se revisó la historia clínica con el fin de precisar antecedentes toxicológicos, sintomatología, tratamiento instaurado, exámenes paraclínicos, diagnóstico y tiempo de permanencia hospitalaria.

Un cuestionario de necropsia verbal fue especialmente diseñado con el objeto de obtener información de las variables ocupación, estrato social, escolaridad, antecedentes toxicológicos que incluían ingesta de alcohol, tipo de licor, sitio habitual de compra de éste, con énfasis particular en tratar de establecer el sitio de aprovisionamiento del licor previo al fallecimiento, antecedentes de consumo de estupefacientes, sintomatologia presentada y atención médica recibida, entre otros. Este cuestionario fue aplicado a los familiares de los fallecidos.

Se revisó el libro de registro del Laboratorio de Toxicología Forense del INML y CF correspondiente al período 1993-1994 y se extractó información relativa a necropsias con muestras positivas para intoxicación por alcohol metílico.
Las muestras de sangre de los casos se procesaron por el método de cromatografía de gases con espaciador de cabeza. Con el objeto de confirmar los resultados, el Laboratorio de Toxicología Forense repitió el análisis de las muestras utilizando el mismo método citado anteriormente (técnica usada desde agosto de 1993) $(8,9)$.

Se definió como caso de intoxicación metílica mortal aquella defunción ocurrida durante junio de 1994, cuya necropsia fue realizada en el Instituto Nacional de Medicina Legal y Ciencias Forenses, Regional Bogotá, con concentraciones de metanol por encima de $30 \mathrm{mg} / \mathrm{dL}$ y cuyo informe de necropsia establecía intoxicación por metanol como causa básica de la defunción; y como caso de indigente a aquella persona que carecía de vivienda durante los 12 meses previos a la defunción y deambulaba por las calles.

Con el objeto de identificar la correlación entre los signos y síntomas y la concentración de etanol y metanol en sangre, se realizó un análisis estadístico utilizando la prueba T para estimar la diferencia de medias de grupos independientes y se calcularon los intervalos de confianza de estas diferencias.

La función estadística scan y las tablas propuestas por S. Wallenstein (10) se utilizaron con el fin de encontrar la probabilidad de ocurrencia al azar de esta conglutinación de casos. 


\section{Resultados}

\section{Tiempo, lugar y persona}

Entre el 1 y el 30 de junio de 1994 se diagnosticaron 14 defunciones por intoxicación con alcohol metílico, mediante la determinación de metanol en sangre realizada en el Laboratorio de Toxicología del INML y CF.

De los 14 casos, 12 fueron llevados a algún centro hospitalario y los dos restantes fueron encontrados muertos por familiares 0 amigos quienes avisaron a la policía y fueron trasladados al INML y CF.

En 6 de los 14 casos identificados no se encontró documento alguno de identidad. La edad tuvo que ser determinada por el patólogo forense de acuerdo con criterios de patología forense (aspecto general, caracteres sexuales secundarios, desarrollo pondoestatural y desgaste óseo y dentario).

En cuanto a la edad, todas las muertes se presentaron en adultos entre los 20 y los 50 años de edad, con un promedio de 36 ; respecto al género, del total de defunciones, 12 se presentaron en hombres y 2 en mujeres.

De los 14 casos identificados durante de junio, tres se presentaron el día 17. No se encontró relación con los días de la semana. La curva epidémica que ilustra su distribución se presenta en la figura 2.

Los casos fueron clasificados en estratos socioeconómicos teniendo en cuenta su sitio de vivienda previo a la pérdida del mismo, el vestido, la ocupación y la información obtenida de las personas que declararon en el proceso. Se estableció que la totalidad de los casos eran indigentes, clasificación que fue corroborada por los dictámenes de necropsia en los cuales se tiene en cuenta la presencia de deterioro del aspecto físico general, desnutrición, desaseo, uñas de manos y pies desquebrajadas y ropa en mal estado.

\section{Manifestaciones clínicas}

La sintomatología referida en las historias clínicas de los 12 pacientes que fueron llevados al hospital fue compatible con intoxicación por alcohol metílico.

El médico de urgencias realizó el diagnóstico clínico de intoxicación por metanol en cuatro de los 12 pacientes que recibieron atención hospitalaria, cinco tuvieron diagnóstico de intoxicación exógena, uno de dolor abdominal en estudio y farmacodependencia, uno de accidente cerebrovascular y uno fue declarado muerto a su arrivo.

En los casos hospitalizados, se observó una rápida evolución de los síntomas neurológicos que los

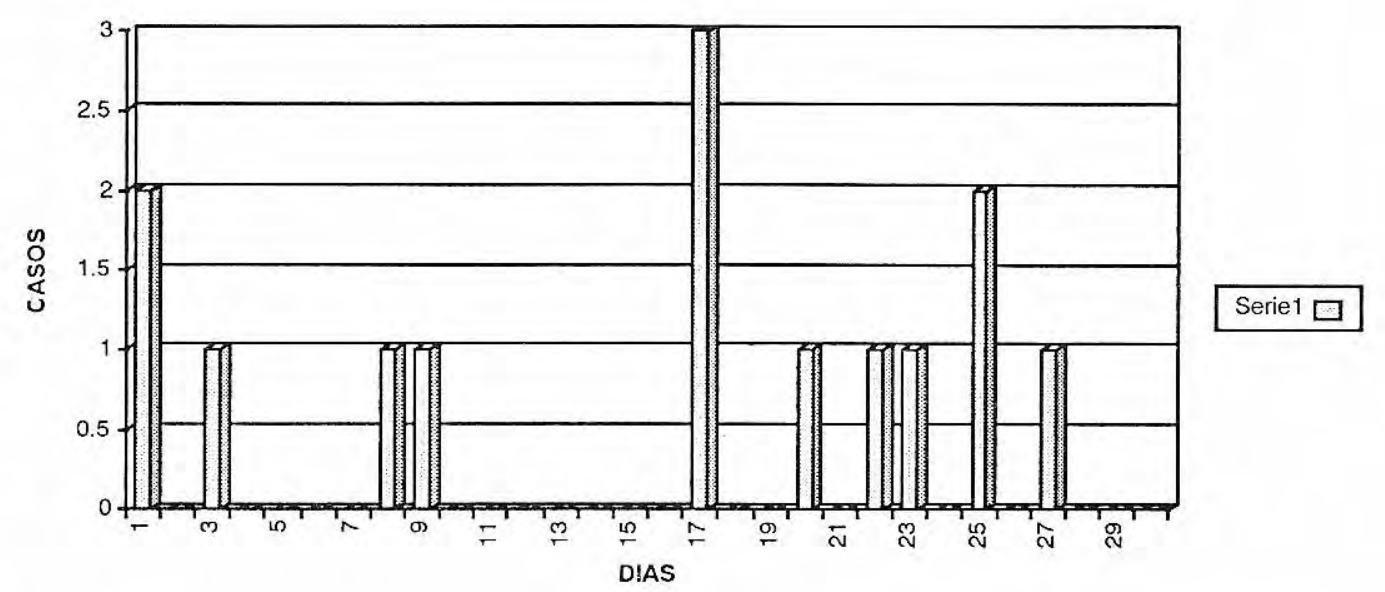

Figura 2. Muertes asociadas con concentraciones elevadas de metanol en sangre por día de fallecimiento, junio de 1994. 
llevó a estupor y coma. Unicamente a un paciente de los cuatro a quienes se les diag-nosticó intoxicación por metanol, se le tomó muestra de sangre para gases arteriales y se encontró severa acidosis metabólica. Dos de los cuatro casos diagnosticados recibieron tra-tamiento con alcohol etílico como antídoto y a ninguno se le realizó hemodiálisis.

El tiempo promedio de supervivencia después de llegar al hospital fue de 6 horas con un rango de 10 minutos a 48 horas.

\section{Antecedentes de ingestión de alcohol y de estupefacientes}

El dato de ingestión de alcohol etílico previa a la intoxicación fue identificado en todas las historias clínicas de los 12 pacientes hospitalizados.

Respecto a las fuentes de consumo de alcohol, se estableció la ingestión previa de alcohol de reverbero o alcohol industrial en dos de los 12 pacientes, aproximadamente de 150 a $300 \mathrm{cc}$. Por otro lado, se encontró que uno de los casos usaba con frecuencia alcohol etílico en fricciones para los dolores articulares.

Con base en los datos de la historia clínica y la necropsia verbal, no se pudo establecer en el resto de los casos, el tipo de licor ingerido, la cantidad, ni el sitio donde fue adquirido. Sin embargo, en 13 de los 14 casos se estableció el antecedente de consumo habitual de metanol en preparaciones o mezclas con bebidas alcohólicas y otras embotelladas.

Se confirmó, además, que seis de los 14 casos tenían antecedentes de consumo de estupefacientes; sólo uno informó antes de fallecer, no tener ningún antecedente y de los siete restantes no se obtuvo información al respecto (cuadro 1).

\section{Determinación de alcohol etílico y metílico}

Todos los casos presentaron concentraciones de metanol en sangre entre 43 y $190,4 \mathrm{mg} / \mathrm{dL}$, con un promedio de $101,9 \mathrm{mg} / \mathrm{dL}$.

No se encontró asociación estadística entre los niveles de metanol en sangre y la sintomatología presentada en los casos hospitalizados, ni con la aparición de manifestaciones graves de intoxicación como ceguera.
Cuadro 1. Antecedentes de uso de tóxicos entre los casos de intoxicación por metanol, Bogotá, junio de 1994.

\begin{tabular}{ll}
\hline Productos & No. \\
\hline Bazuco & 5 \\
Cocaína & 1 \\
Marihuana & 1 \\
Pegantes & 1 \\
\hline
\end{tabular}

Fuente: historias clínicas de los centros hospitalarios.

Las concentraciones de etanol se observaron también elevadas en 10 casos. El rango de las concentraciones fue de 8 a $62,3 \mathrm{mg} / \mathrm{dL}$ con un promedio de $18,2 \mathrm{mg} / \mathrm{dL}$.

Los casos que tuvieron los niveles de etanol más altos, presentaron al mismo tiempo los niveles de metanol más bajos.

\section{Necropsia forense}

Los informes de necropsia confirmaron las secuelas físicas del estado de indigencia de los casos. En seis de los 14 casos, la necropsia se acompañó de examen histopatológico de vísceras y cerebro. Todos los exámenes histopatológicos de pulmón mostraron dilatación e ingurgitación de vasos, fibrosis intersticial y macrófagos pigmentados lo cual es consistente con el uso crónico de bazuco (preparado que resulta de la combinación de residuos del proceso de la obtención de cocaína pura). También se identificó daño hepático centrolobulillar consistente con alcoholismo crónico (11-13).

\section{Necropsia verbal}

Se realizaron seis necropsias verbales a familiares y convivientes. En cuatro de ellas realizadas a los familiares, se pudo establecer que tres de los casos habían cursado hasta quinto de primaria y uno hasta quinto de bachillerato. De las otras dos, cuyos informantes eran convivientes y no familiares, se desconocía la escolaridad.

La edad en la que iniciaron la ingestión de alcohol fue en promedio 17 años con un rango de 13 a 21 años. En seis casos se encontró el antecedente de alcoholismo crónico al igual que el consumo habitual de marihuana, bazuco, cocaína e inhalación de pegantes (Boxer $\left.{ }^{\circledR}\right)$. En tres casos se estableció el uso ocasional de alcohol para fricciones en caso de cefalea o dolor articular. De 
los seis casos en los que se obtuvo una autopsia verbal, sólo en uno se pudo establecer el cuadro clínico que precedió la defunción, el cual correspondía a los síntomas de una intoxicación metílica. No fue posible identificar la fuente de la cual se obtuvo el alcohol consumido durante las 72 horas previas a la defunción en estos seis casos. Se logró establecer en las personas fallecidas que éstas carecían de vivienda durante los 12 meses previos a la defunción. Sin embargo, la necropsia verbal y la verificación de datos realizada en la investigación de campo permitió establecer la zona donde habitualmente permanecían, en todos los casos, dentro del perímetro urbano de Santa Fe de Bogotá.

Se pudo establecer, también, que dos de los 14 casos estaban relacionados ya que eran convivientes. La función estadística scan $(p=0,004)$ indicó que el exceso de casos conglutinados en junio era estadísticamente significativo en relación con el promedio de los 10 meses previos.

\section{Discusión}

La ingestión de alcohol metílico representa una amenaza para la vida, situación que se ha informado sobre todo en poblaciones especiales como alcohólicos, individuos mentalmente enfermos e indigentes que crónicamente lo consumen. El metanol es una sustancia ubicua, de uso industrial, de bajo costo y de venta irrestricta en nuestro país, ya que no existe en la actualidad ningún tipo de control por parte de las autoridades competentes para su adquisicion y uso. Colombia cuenta con un sistema nacional de control de bebidas y licores, el cual puede prevenir intoxicaciones producidas por adulteración por parte del fabricante. Conglomerados de casos como el presente no,son prevenibles por estos mecanismos de inspección sanitaria de alimentos y bebidas. El metanol también es usado comúnmente como combustible en los hogares de escasos recursos. En el país no hay ningún informe sobre la magnitud de este problema, siendo éste el primer estudio de conglutinado de intoxicación por metanol, descrito en la población de indigentes.

La totalidad de los casos era o habían sido 'indigentes lo que sugiere que esta población es de alto riesgo para la intoxicación por metanol. Se estableció que los casos de este brote ingerían frecuentemente metanol y tanto las historias clínicas como las necropsias verbales y los hallazgos histopatológicos son consistentes con la ingestión de alcohol etílico y metílico y con el consumo de bazuco.

Por los informes del INML y CF, la intoxicación por metanol se ha identificado como un problema de endemia silente que cobra, aproximadamente, 50 vidas por año en Santa Fe de Bogotá.

A pesar de que todos los casos tenían el antecedente de ingestión de alcohol, además de los síntomas característicos de intoxicación por alcohol metílico, solo cuatro se beneficiaron del diagnóstico y tan solo a dos de ellos se les dió el antídoto, lo cual era indispensable por la severidad extrema de los casos al igual que el realizar hemodiálisis ya que todos presentaban concentraciones de alcohol metílico en sangre por encima de $30 \mathrm{mg} / \mathrm{dL}$, de acuerdo con las pautas de manejo del paciente intoxicado por alcohol metílico del Hospital San Juan de Dios, Bogotá.

También, fue posible determinar que el personal médico no está lo suficientemente capacitado para la atención del paciente intoxicado, en especial, si se trata del grupo de indigentes.

Una debilidad del estudio es que la necropsia verbal no ayudó a identificar la fuente de aprovisionamiento de alcohol metílico, haciendo imposible la evaluación de hipótesis como la limpieza social o el uso compartido de alcohol industrial.

El presente estudio evidencia la necesidad de: 1) sensibilizar al personal de urgencias en el manejo de indigentes, enfermos mentales y adictos a alcohol y a drogas; 2) entrenar a las personas que laboran en los servicios de urgencias en el diagnóstico y tratamiento adecuado de los casos de intoxicación por metanol; 3) capacitar a los líderes comunitarios de esta población a riesgo (indigentes) con el fin de informarles acerca de los efectos tóxicos producidos por la ingestión de alcohol metílico al igual que a la comunidad en general; 4) mejorar las inspecciones sanitarias con el fin de realizar el adecuado control analítico del 
licor que se expende al público por parte de las instituciones correspondientes; 5) realizar estudios sobre este problema endémico de intoxicación fatal por metanol, que permitan evaluar con mayor precisión su magnitud y los factores de riego que se le asocian.

\section{Referencias}

1. Córdoba D. Toxicología. Segunda edición. Medellín: L. Vieco; 1991.

2. Casarett LJ, Doull CD. Toxicology. 4th ed. Pergamon Press, Inc.; 1991:701-2.

3. Golfranks. Toxicologic emergencies. 4th ed. Appleton \& Lange; 1990:493-7.

4. Bennet IL, Caray FH, Mitchell GL. Acute methyl alcohol poisoning: review based on experiences in outbreak of 323 cases. MedicinE (Baltimore) 1953; 32:431-63.

5. Kang RL, Talbert W, Harlan J, et al. A methanol poisoning outbreak in Kentucky: a clinical epidemiology study. Arch Environ Health 1968; 17:19129.

6. Swarty RD, Millm RP, et al. Epidemic methanol poisoning: clinical and biochemical analysis of a recent episode. Medicine GD 1981;373-82.
7. Guerrero A. Intoxicación masiva por licor adulterado con metanol, Barranquilla, septiembre de 1989. Memorias, Primer Congreso Conjunto Colombiano de Toxicología 1992;109-124.

8. Senkowski C. The accuracy of blood alcohol analysis using headspace gas chromatography when performed on clotted samples. J Forensic Sci 1990;35:170-80.

9. Smith J, Early R. The analysis of blood alcohol using the HP193TA headspace sampler and fused silium capillary colums. Hewlett Packard Aplication Note 228-84; 1989;1-7.

10. Wallenstein $\mathbf{S}$. A test for detection of clustering over time. Am J Epidemiol 1980;11:367-72.

11. Greenebaum E, Copeland A, Grewal R. Blackened bronchoalveolar lavage fluid in crack smokers. A preliminary study: Am J Clin Pathol 1993;100:4817.

12. Forrest JM, Steele AW., Waldron JA., Parson PE. Crack lung: an acute pulmonary syndrome with a spectrum of clinical and histopathologic findings. Am Rev Respir Dis 1990;142:462-7.

13. Anderson WA. Pathology. 6th ed. St. Louis: The C.V. Mosby Company; 1971:1197-1205. 